\title{
]jfis
}

\section{The Sound and Complete Gentzen Deduction System for the Modalized Łukasiewicz Three-Valued Logic}

\section{Cungen Cao and Yuefei Sui}

Key Laboratory of Intelligent Information Processing, Institute of Computing Technology, Chinese Academy of Sciences, Beijing, China

\begin{abstract}
A modalized Łukasiewicz three-valued propositional logic will be proposed in this paper which there are three modalities $[t] ;[m] ;[f]$ to represent the three values $t ; m ; f ;$ respectively. And a Gentzen-typed deduction system will be given so that the the system is sound and complete with respect to the Łukasiewicz three-valued semantics $\bigsqcup_{3}$, which are given in soundness theorem and completeness theorem.
\end{abstract}

Keywords: Łukasiewicz three-valued logic, Modality, Soundness, Completeness

\section{Introduction}

The three-valued logics are traditional and have been studied in variant ways ([4-7, 14]). There are the following three-valued logics:

- Bochvar's three-valued logic ( [3, 6]), which logical language contains the logical connectives: $\vee, \wedge, \rightarrow, \leftrightarrow, \equiv,=$, and the following semantics:

\begin{tabular}{c|rcr|rrr|rrr}
\multicolumn{3}{r|}{} & \multicolumn{3}{|c|}{$p \wedge q$} & \multicolumn{3}{c|}{$p \vee q$} & \multicolumn{3}{c}{$p \rightarrow q$} \\
\hline$p$ & 1 & 0 & -1 & 1 & 0 & -1 & 1 & 0 & -1 \\
\hline 1 & 1 & 0 & -1 & 1 & 0 & 1 & 1 & 0 & -1 \\
0 & 0 & 0 & 0 & 0 & 0 & 0 & 0 & 0 & 0 \\
-1 & -1 & 0 & -1 & 1 & 0 & -1 & 1 & 0 & 1
\end{tabular}

Received: Jul. 17, 2015

Revised : Sep. 21, 2016

Accepted: Sep. 22, 2016

Correspondence to: Corresponding author (email@address)

(CThe Korean Institute of Intelligent Systems

(C)This is an Open Access article distributed under the terms of the Creative Commons Attribution Non-Commercial License (http://creativecommons.org/licenses/ by-nc/3.0// which permits unrestricted noncommercial use, distribution, and reproduction in any medium, provided the original work is properly cited.

\begin{tabular}{c|rrr|rrr|rrr} 
& \multicolumn{3}{|c|}{$p \leftrightarrow q$} & \multicolumn{3}{|c|}{$p \equiv q$} & \multicolumn{3}{c}{$p=q$} \\
$p q$ & 1 & 0 & -1 & 1 & 0 & -1 & 1 & 0 & -1 \\
\hline 1 & 1 & 0 & -1 & 1 & -1 & -1 & 1 & -1 & -1 \\
0 & 0 & 0 & 0 & -1 & 1 & 1 & -1 & 1 & -1 \\
-1 & -1 & 0 & 1 & -1 & 1 & 1 & -1 & -1 & 1
\end{tabular}

- Kleene's three-valued logic ( [8, 14]), which logical language contains the logical connectives: $\neg, \vee, \wedge, \rightarrow$, and the following semantics:

\begin{tabular}{c|c|ccc|ccc|ccc} 
& $\neg p$ & \multicolumn{3}{|c|}{$p \wedge q$} & \multicolumn{3}{c|}{$p \vee q$} & \multicolumn{3}{c}{$p \rightarrow q$} \\
\hline \multicolumn{1}{r|}{$q$} & & 1 & 0 & -1 & 1 & 0 & -1 & 1 & 0 & -1 \\
\hline 1 & -1 & 1 & 0 & -1 & 1 & 1 & 1 & 1 & 0 & -1 \\
0 & 0 & 0 & 0 & -1 & 1 & 0 & 0 & 1 & 0 & 0 \\
-1 & 1 & 1 & -1 & -1 & 1 & 0 & -1 & 1 & 1 & 1
\end{tabular}


- Łukasiewicz's three-valued logic ( [10, 11]), which logical language contains the logical connectives: $\neg, \mathbf{M}, \mathbf{L}, \mathbf{I}, \vee, \wedge, \prec$ , $\equiv$, and the following semantics:

\begin{tabular}{c|cccc}
$p$ & $\neg p$ & $\mathbf{M} p$ & $\mathbf{L} p$ & $\mathbf{I} p$ \\
\hline $\mathrm{t}$ & $\mathrm{f}$ & $\mathrm{t}$ & $\mathrm{t}$ & $\mathrm{f}$ \\
$\mathrm{m}$ & $\mathrm{m}$ & $\mathrm{t}$ & $\mathrm{f}$ & $\mathrm{t}$ \\
$\mathrm{f}$ & $\mathrm{t}$ & $\mathrm{f}$ & $\mathrm{f}$ & $\mathrm{f}$
\end{tabular}

and

\begin{tabular}{c|ccc|ccc|ccc|cccc}
\multicolumn{1}{c|}{} & \multicolumn{3}{|c|}{$p \wedge q$} & \multicolumn{3}{c|}{$p \vee q$} & \multicolumn{3}{c|}{$p \prec q$} & \multicolumn{3}{|c}{$p \equiv q$} \\
\hline $\mathrm{f}$ & $\mathrm{t}$ & $\mathrm{m}$ & $\mathrm{f}$ & $\mathrm{t}$ & $\mathrm{m}$ & $\mathrm{f}$ & $\mathrm{t}$ & $\mathrm{m}$ & $\mathrm{f}$ & $\mathrm{t}$ & $\mathrm{m}$ & $\mathrm{f}$ \\
$\mathrm{t}$ & $\mathrm{t}$ & $\mathrm{m}$ & $\mathrm{f}$ & $\mathrm{t}$ & $\mathrm{t}$ & $\mathrm{t}$ & $\mathrm{t}$ & $\mathrm{m}$ & $\mathrm{f}$ & $\mathrm{t}$ & $\mathrm{m}$ & $\mathrm{f}$ \\
$\mathrm{m}$ & $\mathrm{m}$ & $\mathrm{m}$ & $\mathrm{f}$ & $\mathrm{t}$ & $\mathrm{m}$ & $\mathrm{m}$ & $\mathrm{t}$ & $\mathrm{t}$ & $\mathrm{m}$ & $\mathrm{m}$ & $\mathrm{t}$ & $\mathrm{m}$ \\
$\mathrm{f}$ & $\mathrm{f}$ & $\mathrm{f}$ & $\mathrm{f}$ & $\mathrm{t}$ & $\mathrm{m}$ & $\mathrm{f}$ & $\mathrm{t}$ & $\mathrm{t}$ & $\mathrm{t}$ & $\mathrm{f}$ & $\mathrm{m}$ & $\mathrm{t}$
\end{tabular}

- Post's three-valued logic ([12, 13]), which logical language contains the logical connectives: $\neg, \vee, \wedge, \rightarrow, \leftrightarrow$, and the following semantics:

\begin{tabular}{|c|c|c|c|c|c|c|c|c|c|c|c|c|c|c|}
\hline & $\neg p$ & \multicolumn{3}{|c|}{$p \vee q$} & \multicolumn{3}{|c|}{$p \wedge q$} & \multicolumn{4}{|c|}{$p \rightarrow q$} & \multicolumn{3}{|c|}{$p \leftrightarrow q$} \\
\hline & & & $\mathrm{m}$ & & & n & $\mathrm{f}$ & & $=$ & n & & & m & $\mathrm{f}$ \\
\hline t & $\mathrm{f}$ & & $t$ & & & f & $\mathrm{m}$ & & $=$ & n & & & $f$ & \\
\hline $\mathrm{m}$ & $\mathrm{m}$ & & $\mathrm{m}$ & $\mathrm{m}$ & & & $\mathrm{m}$ & & $=$ & n & $\mathbb{E}$ & & $t$ & \\
\hline$f$ & t & t & $\mathrm{m}$ & $\mathrm{f}$ & $\mathrm{m}$ & $\mathrm{m}$ & $\mathrm{m}$ & & $=t$ & t & E & f & $\mathrm{m}$ & \\
\hline
\end{tabular}

In such logics, the negation $\neg$ is contrary, not contradictory as in the traditional two-valued logics. Avris ( [1,2]) gave a sound and complete Gentzen deduction system for such three-valued logics in which the intermediate value $m$ has no contribution to the deduction, so that the system for the three-valued logics works for the four-valued logics, the five-valued logics, and so on.

Zhu ( [15-17]) proposed a three-valued logic, called the intermediate logic, in which a creative unary logical connective $\sim$ is given such that $\sim A$ is true if and only if the truth-value of $A$ is $\mathrm{m}$, which makes the intermediate logic have different Gentzen deduction system from the traditional one ( [9]).

To represent the intermediate values in the logic, we should introduce unary modalities $[\mathrm{t}],[\mathrm{m}],[\mathrm{f}]$, so that $[\mathrm{t}] A$ is true iff $A$ is true; $[\mathrm{m}] A$ is true iff $A$ is intermediate; and [f] $A$ is true iff $A$ is false. Hence, we shall give a modalized propositional logic which logical language contains the following logical symbols:

- the unary connectives: $\neg, \mathbf{M}, \mathbf{L}, \mathbf{I}$;

- the unary modalites: $[\mathrm{t}],[\mathrm{m}],[\mathrm{f}]$; and

- the binary connectives: $\wedge, \vee, \prec, \equiv$.
We will give a sound and complete Gentzen deduction system for Łukasiewicz's three-valued propositional logic, that is, for any sequent $\Gamma \Rightarrow \Delta$,

- The soundness theorem: If $\vdash_{\biguplus_{3}} \Gamma \Rightarrow \Delta$ then $\Gamma \models_{\mathfrak{Ł}_{3}} \Delta$.

- The completeness theorem: If $\models_{\mathfrak{Ł}_{3}} \Gamma \Rightarrow \Delta$ then $\Gamma \vdash_{\mathfrak{Ł}_{3}} \Delta$.

This paper is organized as follows: the next section defines the basic elements in Lukasiewicz's three-valued logic: the logical language, syntax and semantics; Section 3 gives a deduction system for Łukasiewicz's three-valued propositional logic and proves the soundness theorem; Section 4 proves the completeness theorem for Łukasiewicz's three-valued propositional logic, and Section 5 concludes the whole paper.

Our notation is standard, and a reference is [9].

\section{The Modalized Lukasiewicz Three-Valued Propositional Logic}

Let the logical language contain the following symbols:

- propositional variables: $p_{0}, p_{1}, \ldots$;

- modalities: $[\mathrm{t}],[\mathrm{m}],[\mathrm{f}]$,

- unary logical connectives: $\neg, \mathbf{M}, \mathbf{L}, \mathbf{I}$, and

- binary logical connectives: $\wedge, \vee, \prec, \equiv$.

Formulas:

$$
\begin{array}{rlrl}
A::= & p & & \text { (atomic) } \\
& \left|[\mathrm{t}] A_{1}\right|[\mathrm{m}] A_{1} \mid[\mathbf{f}] A_{1} & & \text { (modalized) } \\
& \left|\neg A_{1}\right| \mathbf{M} A_{1}\left|\mathbf{L} A_{1}\right| \mathbf{I} \mathbf{A}_{1} & & \text { (unary connective) } \\
& \left|A_{1} \wedge A_{2}\right| A_{1} \vee A_{2} \mid A_{1} & \\
& \prec A_{2} \mid A_{1} \equiv A_{2} & & \text { (binary connective) }
\end{array}
$$

Let $v$ be a function from the propositional variables to $\mathrm{Ł}_{3}=$ $(\{\mathrm{t}, \mathrm{m}, \mathrm{f}\}, \leq)$.

Define

$$
v(A)= \begin{cases}v(p) & \text { if } A=p \\ g_{*}\left(v\left(A_{1}\right)\right) & \text { if } A=[*] A_{1} \\ f_{\diamond}\left(v\left(A_{1}\right)\right) & \text { if } A=\diamond A_{1} \\ h_{\bullet}\left(v\left(A_{1}\right), v\left(A_{2}\right)\right) & \text { if } A=A_{1} \bullet A_{2},\end{cases}
$$

where $* \in\{\mathrm{t}, \mathrm{m}, \mathrm{f}\}, \diamond \in\{\neg, \mathbf{M}, \mathbf{L}, \mathbf{I}\}, \bullet \in\{\wedge, \vee, \prec, \equiv\}$ and

\begin{tabular}{c|cccc|cccc} 
& $g_{\mathrm{t}}$ & $g_{\mathrm{m}}$ & $g_{\mathrm{f}}$ & & $f_{\neg}$ & $f_{\mathbf{M}}$ & $f_{\mathbf{L}}$ & $f_{\mathbf{I}}$ \\
\hline $\mathrm{t}$ & $\mathrm{t}$ & $\mathrm{f}$ & $\mathrm{m}$ & $\mathrm{t}$ & $\mathrm{f}$ & $\mathrm{t}$ & $\mathrm{t}$ & $\mathrm{f}$ \\
$\mathrm{m}$ & $\mathrm{m}$ & $\mathrm{t}$ & $\mathrm{f}$ & $\mathrm{m}$ & $\mathrm{m}$ & $\mathrm{t}$ & $\mathrm{f}$ & $\mathrm{t}$ \\
$\mathrm{f}$ & $\mathrm{f}$ & $\mathrm{m}$ & $\mathrm{t}$ & $\mathrm{f}$ & $\mathrm{t}$ & $\mathrm{f}$ & $\mathrm{f}$ & $\mathrm{f}$
\end{tabular}

and 


\begin{tabular}{|c|c|c|c|c|c|c|c|c|c|c|c|c|}
\hline & \multicolumn{3}{|c|}{$h_{\wedge}$} & \multicolumn{3}{|c|}{$h_{\vee}$} & \multicolumn{3}{|c|}{$h_{\prec}$} & \multicolumn{3}{|c|}{$h_{\equiv}$} \\
\hline & $t$ & $\mathrm{~m}$ & $\mathrm{f}$ & $t$ & $\mathrm{~m}$ & $\mathrm{f}$ & $t$ & $\mathrm{~m}$ & $\mathrm{f}$ & $t$ & $\mathrm{~m}$ & $\mathrm{f}$ \\
\hline$t$ & $t$ & $\mathrm{~m}$ & $\mathrm{f}$ & $t$ & $t$ & $t$ & $t$ & $\mathrm{~m}$ & $f$ & t & $\mathrm{m}$ & $\mathrm{f}$ \\
\hline $\mathrm{m}$ & $\mathrm{m}$ & $\mathrm{m}$ & $\mathrm{f}$ & $t$ & $\mathrm{~m}$ & $\mathrm{~m}$ & $t$ & $t$ & $\mathrm{~m}$ & m & $t$ & $\mathrm{~m}$ \\
\hline $\mathrm{f}$ & $\mathrm{f}$ & $\mathrm{f}$ & $\mathrm{f}$ & $t$ & $\mathrm{~m}$ & $\mathrm{f}$ & $t$ & $t$ & $t$ & $\mathrm{f}$ & $\mathrm{m}$ & $t$ \\
\hline
\end{tabular}

Given two sets $\Gamma, \Delta$ of formulas, define

$$
\begin{aligned}
& v(\Gamma)=\min \{v(A): A \in \Gamma\}, \\
& v(\Delta)=\max \{v(A): A \in \Delta\},
\end{aligned}
$$

where the $\leq$-relation is that of $\succeq_{3}$.

Given a sequent $\delta=\Gamma \Rightarrow \Delta$, we say that $v$ satisfies $\delta$, denoted by $v \models_{\mathfrak{七}_{3}} \delta$, if $v(\Gamma) \leq v(\Delta)$.

A sequent $\delta$ is valid, denoted by $\models_{\mathrm{七}_{3}} \delta$, if for any assignment $v, v \models \mathfrak{七}_{3} \delta$.

Assume that $[\mathrm{t}] p,[\mathrm{~m}] p,[\mathrm{f}] p$ corresponds to the three values: $+1,0,-1$, respectively. Then, we have

$$
\begin{aligned}
& {[\mathrm{t}](A \wedge B)=[\mathrm{t}] A \wedge[\mathrm{t}] B} \\
& {[\mathrm{~m}](A \wedge B)=[\mathrm{t}] A[\mathrm{~m}] B \underline{\vee}[\mathrm{m}] A[\mathrm{t}] B \underline{\vee}[\mathrm{m}] A[\mathrm{~m}] B} \\
& \underline{\vee}[\mathrm{m}] A[\mathrm{f}] B \underline{\vee}[\mathrm{f}] A[\mathrm{~m}] B \\
& {[\mathrm{f}](A \wedge B)=[\mathrm{t}] A[\mathrm{f}] B \underline{\vee}[\mathrm{f}] A[\mathrm{t}] B \underline{\vee}[\mathrm{f}] A[\mathrm{f}] B ;} \\
& {[\mathrm{t}](A \vee B)=[\mathrm{t}] A[\mathrm{t}] B \underline{\vee}[\mathrm{t}] A[\mathrm{f}] B \underline{\vee}[\mathrm{f}] A[\mathrm{t}] B} \\
& {[\mathrm{~m}](A \vee B)=[\mathrm{m}] A[\mathrm{~m}] B \underline{\vee}[\mathrm{m}] A[\mathrm{f}] B \underline{\vee}[\mathrm{f}] A[\mathrm{~m}] B} \\
& \underline{\mathrm{V}}[\mathrm{t}] A[\mathrm{~m}] \underline{\mathrm{V}}[\mathrm{m}] A[\mathrm{t}] B \\
& {[\mathrm{f}](A \vee B)=[\mathrm{f}] A[\mathrm{f}] B \text {, }}
\end{aligned}
$$

where $\underline{\Lambda}, \underline{\vee}$ denote $\wedge, \vee$ in the two-valued propositional logic, respectively, and $A B$ denotes $A \triangle B$. Similarly we have the equivalences for $\rightarrow, \prec, \equiv$ and $=$.

\section{The Gentzen Deduction System}

The Gentzen deduction system contains the following axioms and deduction rules.

- Axioms:

$$
\begin{array}{ll}
\Gamma,[*] p \Rightarrow[*] p, \Delta ; & \Gamma,\left[*_{1}\right] A,\left[*_{2}\right] A \Rightarrow \Delta ; \\
\Gamma,[\diamond] p \Rightarrow[\diamond] p, \Delta, & \Gamma,\left[\diamond_{1}\right] A,\left[\diamond_{2}\right] A \Rightarrow \Delta, \\
\Gamma \Rightarrow[\mathrm{t}] p,[\mathrm{~m}] p,[\mathrm{f}] p, \Delta, &
\end{array}
$$

where $*_{1} \neq *_{2}, \diamond_{1} \neq \diamond_{2}$.

- The deduction rules for modalities:

$$
\frac{\Gamma,[\mathrm{t}] A \Rightarrow \Delta}{\Gamma,[\mathrm{t}][\mathrm{t}] A \Rightarrow \Delta}\left([\mathrm{t}][\mathrm{t}]^{L}\right) \quad \frac{\Gamma,[\mathrm{m}] A \Rightarrow \Delta}{\Gamma,[\mathrm{t}][\mathrm{m}] A \Rightarrow \Delta}\left([\mathrm{t}][\mathrm{m}]^{L}\right)
$$

$$
\begin{aligned}
& \frac{\Gamma,[\mathrm{f}] A \Rightarrow \Delta}{\Gamma,[\mathrm{t}][\mathrm{f}] A \Rightarrow \Delta}\left([\mathrm{t}][\mathrm{f}]^{L}\right) \\
& \overline{\Gamma,[\mathrm{m}][\mathrm{t}] A \Rightarrow \Delta}\left([\mathrm{m}][\mathrm{t}]^{L}\right) \quad \overline{\Gamma,[\mathrm{m}][\mathrm{m}] A \Rightarrow \Delta}\left([\mathrm{m}][\mathrm{m}]^{L}\right) \\
& \overline{\Gamma,[\mathrm{m}][\mathrm{f}] A \Rightarrow \Delta}\left([\mathrm{m}][\mathrm{f}]^{L}\right) \\
& \frac{\Gamma,[\mathrm{f}] A \underline{\bigvee}[\mathrm{m}] A \Rightarrow \Delta}{\Gamma,[\mathrm{f}][\mathrm{t}] A \Rightarrow \Delta}\left([\mathrm{f}][\mathrm{t}]^{L}\right) \\
& \frac{\Gamma,[\mathrm{t}] A \underline{\bigvee}[\mathrm{f}] A \Rightarrow \Delta}{\Gamma,[\mathrm{f}][\mathrm{m}] A \Rightarrow \Delta}\left([\mathrm{f}][\mathrm{m}]^{L}\right) \\
& \frac{\Gamma,[\mathrm{t}] A \underline{\vee}[\mathrm{m}] \Rightarrow \Delta}{\Gamma,[\mathrm{f}][\mathrm{f}] A \Rightarrow \Delta}\left([\mathrm{f}][\mathrm{f}]^{L}\right)
\end{aligned}
$$

Here, for the simplicity, we miss the deduction rules of the right side, and the same for the following rules for the unary logical connectives.

\section{- The deduction rules for unary logical connectives:}

$$
\begin{aligned}
& \frac{\Gamma, A \Rightarrow \Delta}{\Gamma, \neg \neg A \Rightarrow \Delta}\left(\neg \neg^{L}\right) \quad \frac{\Gamma,[\mathbf{f}] A \Rightarrow \Delta}{\Gamma, \neg \mathbf{M} A \Rightarrow \Delta}\left(\neg \mathbf{M}^{L}\right) \\
& \frac{\Gamma,[\mathrm{m}] A \underline{\vee}[\mathrm{f}] A \Rightarrow \Delta}{\Gamma, \neg \mathbf{L} A \Rightarrow \Delta}\left(\neg \mathbf{L}^{L}\right) \quad \frac{\Gamma,[\mathrm{t}] A \underline{\vee}[\mathrm{f}] A \Rightarrow \Delta}{\Gamma, \neg \mathbf{I} A \Rightarrow \Delta}\left(\neg \mathbf{I}^{L}\right) \\
& \frac{\Gamma,[\mathrm{m}] A \underline{\mathrm{V}}[\mathrm{f}] A \Rightarrow \Delta}{\Gamma, \mathbf{M} \neg A \Rightarrow \Delta}\left(\mathbf{M}^{L}{ }^{L}\right) \quad \frac{\Gamma,[\mathrm{t}] A \Rightarrow \Delta}{\Gamma, \mathbf{M M} A \Rightarrow \Delta}\left(\mathbf{M M}^{L}\right) \\
& \frac{\Gamma,[\mathrm{t}] A \Rightarrow \Delta}{\Gamma, \mathbf{M L} A \Rightarrow \Delta}\left(\mathbf{M L}^{L}\right) \quad \frac{\Gamma,[\mathrm{m}] A \Rightarrow \Delta}{\Gamma, \mathbf{M I} A \Rightarrow \Delta}\left(\mathbf{M I}^{L}\right) \\
& \frac{\Gamma,[\mathbf{f}] A \Rightarrow \Delta}{\Gamma, \mathbf{L} \neg A \Rightarrow \Delta}\left(\mathbf{L} \neg^{L}\right) \quad \frac{\Gamma,[\mathrm{t}] A \underline{\vee}[\mathrm{m}] A \Rightarrow \Delta}{\Gamma, \mathbf{L M} A \Rightarrow \Delta}\left(\mathbf{L M}^{L}\right) \\
& \frac{\Gamma,[\mathrm{t}] A \Rightarrow \Delta}{\Gamma, \mathbf{L} \mathbf{L} A \Rightarrow \Delta}\left(\mathbf{L L}^{L}\right) \quad \frac{\Gamma,[\mathrm{m}] A \Rightarrow \Delta}{\Gamma, \mathbf{L I} A \Rightarrow \Delta}\left(\mathbf{L I}^{L}\right) \\
& \frac{\Gamma,[\mathrm{m}] A \Rightarrow \Delta}{\Gamma, \mathbf{I} \neg A \Rightarrow \Delta}\left(\mathbf{I} \neg^{L}\right) \quad \overline{\Gamma, \mathbf{I M} A \Rightarrow \Delta}\left(\mathbf{I M}^{L}\right) \\
& \overline{\Gamma, \mathbf{I L} A \Rightarrow \Delta}\left(\mathbf{I L}^{L}\right) \quad \overline{\Gamma, \mathbf{I I} A \Rightarrow \Delta}\left(\mathbf{I I}^{L}\right)
\end{aligned}
$$

- The deduction rules for modalities and unary logical connectives:

$$
\begin{array}{ll}
\frac{\Gamma,[\mathrm{f}] A \Rightarrow \Delta}{\Gamma,[\mathrm{t}] \neg A \Rightarrow \Delta}\left([\mathrm{t}] \neg^{L}\right) & \frac{\Gamma,[\mathrm{t}] A \underline{\bigvee}[\mathrm{m}] \Rightarrow \Delta}{\Gamma,[\mathrm{t}] \mathbf{M} A \Rightarrow \Delta}\left([\mathrm{t}] \mathbf{M}^{L}\right) \\
\frac{\Gamma,[\mathrm{t}] A \Rightarrow \Delta}{\Gamma,[\mathrm{t}] \mathbf{L} A \Rightarrow \Delta}\left([\mathrm{t}] \mathbf{L}^{L}\right) & \frac{\Gamma,[\mathrm{m}] A \Rightarrow \Delta}{\Gamma,[\mathrm{t}] \mathbf{I} A \Rightarrow \Delta}\left([\mathrm{t}] \mathbf{I}^{L}\right) \\
\frac{\Gamma,[\mathrm{m}] A \Rightarrow \Delta}{\Gamma,[\mathrm{m}] \neg A \Rightarrow \Delta}\left([\mathrm{m}] \neg^{L}\right) & \\
\frac{\Gamma,[\mathrm{t}] A \Rightarrow \Delta}{\Gamma,[\mathrm{f}] \neg A \Rightarrow \Delta}\left([\mathrm{f}] \neg^{L}\right) & \frac{\Gamma,[\mathrm{f}] A \Rightarrow \Delta}{\Gamma,[\mathrm{f}] \mathbf{M} A \Rightarrow \Delta}\left([\mathrm{f}] \mathbf{M}^{L}\right) \\
\frac{\Gamma,[\mathrm{m}] A \underline{\bigvee}[\mathrm{f}] A \Rightarrow \Delta}{\Gamma,[\mathrm{f}] \mathbf{L} A \Rightarrow \Delta}\left([\mathrm{f}] \mathbf{L}^{L}\right) & \frac{\Gamma,[\mathrm{t}] A \underline{\bigvee}[\mathrm{f}] A \Rightarrow \Delta}{\Gamma,[\mathbf{f}] \mathbf{I} A \Rightarrow \Delta}\left([\mathbf{f}] \mathbf{I}^{L}\right)
\end{array}
$$

and

$$
\frac{\Gamma,[\mathrm{f}] A \Rightarrow \Delta}{\Gamma, \neg[\mathrm{t}] A \Rightarrow \Delta}\left(\neg[\mathrm{t}]^{L}\right) \quad \frac{\Gamma,[\mathrm{t}] A \Rightarrow \Delta}{\Gamma, \neg[\mathrm{m}] A \Rightarrow \Delta}\left(\neg[\mathrm{m}]^{L}\right)
$$


$\frac{\Gamma,[\mathrm{m}] A \Rightarrow \Delta}{\Gamma, \neg[\mathrm{f}] A \Rightarrow \Delta}\left(\neg[\mathrm{f}]^{L}\right)$

$\frac{\Gamma,[\mathrm{t}] A \underline{\mathrm{\vee}}[\mathrm{f}] A \Rightarrow \Delta}{\Gamma, \mathbf{M}[\mathrm{t}] A \Rightarrow \Delta}\left(\mathbf{M}[\mathrm{t}]^{L}\right)$

$\frac{\Gamma,[\mathrm{m}] A \underline{\bigvee}[\mathrm{f}] A \Rightarrow \Delta}{\Gamma, \mathbf{M}[\mathrm{m}] A \Rightarrow \Delta}\left(\mathbf{M}[\mathrm{m}]^{L}\right)$

$\frac{\Gamma,[\mathrm{t}] A \underline{\mathrm{V}}[\mathrm{f}] A \Rightarrow \Delta}{\Gamma, \mathbf{M}[\mathrm{f}] A \Rightarrow \Delta}\left(\mathbf{M}[\mathrm{f}]^{L}\right)$

$\frac{\Gamma,[\mathrm{t}] A \Rightarrow \Delta}{\Gamma, \mathbf{L}[\mathrm{t}] A \Rightarrow \Delta}\left(\mathbf{L}[\mathrm{t}]^{L}\right)$

$\frac{\Gamma,[\mathrm{m}] A \Rightarrow \Delta}{\Gamma, \mathbf{L}[\mathrm{m}] A \Rightarrow \Delta}\left(\mathbf{L}[\mathrm{m}]^{L}\right)$

$\frac{\Gamma,[\mathbf{f}] A \Rightarrow \Delta}{\Gamma, \mathbf{L}[\mathbf{f}] A \Rightarrow \Delta}\left(\mathbf{L}[\mathbf{f}]^{L}\right)$

$\frac{\Gamma,[\mathrm{m}] A \Rightarrow \Delta}{\Gamma, \mathbf{I}[\mathrm{t}] A \Rightarrow \Delta}\left(\mathbf{I}[\mathrm{t}]^{L}\right)$

$\frac{\Gamma,[\mathrm{f}] A \Rightarrow \Delta}{\Gamma, \mathbf{I}[\mathrm{m}] A \Rightarrow \Delta}\left(\mathbf{I}[\mathrm{m}]^{L}\right)$

$\frac{\Gamma,[\mathrm{t}] A \Rightarrow \Delta}{\Gamma, \mathbf{I}[\mathrm{f}] A \Rightarrow \Delta}\left(\mathbf{I}[\mathbf{f}]^{L}\right)$

\section{- The deduction rules for binary logical connectives:}

$\frac{\Gamma, A, B \Rightarrow \Delta}{\Gamma, A \wedge B \Rightarrow \Delta}\left(\wedge^{L}\right) \frac{\Gamma \Rightarrow A, \Delta \Gamma \Rightarrow B, \Delta}{\Gamma \Rightarrow A \wedge B, \Delta}\left(\wedge^{R}\right)$

$\frac{\Gamma, A \Rightarrow \Delta \Gamma, B \Rightarrow \Delta}{\Gamma, A \vee B \Rightarrow \Delta}\left(\vee^{L}\right) \frac{\Gamma \Rightarrow A, B, \Delta}{\Gamma \Rightarrow A \vee B, \Delta}\left(\vee^{R}\right)$

$\Gamma, A, B \Rightarrow \Delta \Gamma,[\mathrm{m}] A,[\mathrm{t}] B \Rightarrow \Delta \Gamma,[\mathrm{m}] A,[\mathrm{~m}] B$

$$
\Rightarrow \Delta
$$

$\Gamma,[\mathrm{f}] A,[\mathrm{t}] B \Rightarrow \Delta \Gamma,[\mathrm{f}] A,[\mathrm{~m}] B$

$$
\Rightarrow \Delta \Gamma,[\mathrm{f}] A,[\mathrm{f}] B \Rightarrow \Delta
$$

$\Gamma, A \prec B \Rightarrow \Delta$

$\Gamma \Rightarrow A \triangle B,[\mathrm{~m}] A \triangle[\mathrm{t}] B,[\mathrm{~m}] A \triangle[\mathrm{m}] B$,

$\frac{[\mathrm{f}] A \triangleq[\mathrm{t}] B,[\mathrm{f}] A \wedge[\mathrm{m}] B,[\mathrm{f}] A \underline{\mathrm{f}]}[\overline{\mathrm{f}}, \Delta}{\Gamma \Rightarrow A \prec B, \Delta}\left(\prec^{R}\right)$

$\frac{\Gamma, A, B \Rightarrow \Delta \Gamma,[\mathrm{m}] A,[\mathrm{~m}] B \Rightarrow \Delta \Gamma,[\mathrm{f}] A,[\mathrm{f}] B \Rightarrow \Delta}{\Gamma, A \equiv B \Rightarrow \Delta}\left(\equiv^{L}\right)$

$\frac{\Gamma \Rightarrow A \wedge B,[\mathrm{~m}] A \wedge[\mathrm{m}] B,[\mathrm{f}] A \wedge[\mathrm{f}] B, \Delta}{\Gamma \Rightarrow A \equiv B, \Delta}\left(\equiv^{R}\right)$

- The deduction rules for modalities and binary logical connectives:

$\diamond[t]+\diamond:$ the same as the ones for logical connectives.

$\diamond[\mathrm{m}]+\diamond:$

$\Gamma,[\mathrm{t}] A,[\mathrm{~m}] B \Rightarrow \Delta \Gamma_{2},[\mathrm{~m}] A,[\mathrm{t}] B$

$\frac{\Rightarrow \Delta \Gamma,[\mathrm{m}] A,[\mathrm{~m}] B \Rightarrow \Delta}{\Gamma,[\mathrm{m}](A \wedge B) \Rightarrow \Delta}\left([\mathrm{m}] \wedge^{L}\right)$

$\frac{\Gamma \Rightarrow[\mathrm{t}] A \wedge[\mathrm{m}] B,[\mathrm{~m}] A \wedge[\mathrm{t}] B,[\mathrm{~m}] A \wedge[\mathrm{m}] B, \Delta}{\Gamma \Rightarrow[\mathrm{m}](A \wedge B), \Delta}\left([\mathrm{m}] \wedge^{R}\right)$
$\Gamma,[\mathrm{m}] A,[\mathrm{~m}] B \Rightarrow \Delta \Gamma,[\mathrm{m}] A,[\mathrm{f}] B$

$\Rightarrow \Delta \Gamma,[\mathrm{f}] A,[\mathrm{~m}] B \Rightarrow \Delta$
$\Gamma,[\mathrm{m}](A \vee B) \Rightarrow \Delta$
$\frac{\left.\Gamma \Rightarrow[\mathrm{m}] \vee^{L}\right)}{\Gamma \unrhd[\mathrm{m}] B,[\mathrm{~m}] A \wedge[\mathrm{f}] B,[\mathrm{f}] A \wedge[\mathrm{m}] B, \Delta}\left([\mathrm{m}] \vee^{R}\right)$
$\frac{\Gamma,[\mathrm{t}] A,[\mathrm{~m}] B \Rightarrow \Delta \Gamma,[\mathrm{m}] A,[\mathrm{f}] B \Rightarrow \Delta}{\Gamma,[\mathrm{m}](A \prec B) \Rightarrow \Delta}\left([\mathrm{m}] \prec^{L}\right)$
$\frac{\Gamma \Rightarrow[\mathrm{t}] A \wedge[\mathrm{m}] B,[\mathrm{~m}] A \wedge[\mathrm{f}] B, \Delta}{\Gamma \Rightarrow[\mathrm{m}](A \prec B), \Delta}\left([\mathrm{m}] \prec^{R}\right)$
$\Gamma,[\mathrm{t}] A,[\mathrm{~m}] B \Rightarrow \Delta \Gamma,[\mathrm{m}] A,[\mathrm{t}] B \Rightarrow \Delta \Gamma,[\mathrm{m}] A,[\mathrm{f}] B$
$\Rightarrow \Delta \Gamma,[\mathrm{f}] A,[\mathrm{~m}] B \Rightarrow \Delta$
$\Gamma,[\mathrm{m}](A \equiv B) \Rightarrow \Delta$

$\Gamma \Rightarrow[\mathrm{t}] A \wedge[\mathrm{m}] B,[\mathrm{~m}] A \wedge[\mathrm{t}] B,[\mathrm{~m}] A$

$\stackrel{\wedge[\mathrm{f}] B,[\mathrm{f}] A \wedge[\mathrm{m}] B, \Delta}{\Gamma \Rightarrow[\mathrm{m}](A \equiv B), \Delta}\left([\mathrm{m}] \equiv^{R}\right)$

$\diamond[\mathrm{f}]+\diamond:$

$\frac{\Gamma,[\mathrm{f}] A \Rightarrow \Delta \Gamma,[\mathrm{f}] B \Rightarrow \Delta}{\Gamma,[\mathrm{f}](A \wedge B) \Rightarrow \Delta}\left([\mathrm{f}] \wedge^{L}\right)$

$\frac{\Gamma \Rightarrow[\mathbf{f}] A,[\mathbf{f}] B, \Delta}{\Gamma \Rightarrow[\mathbf{f}](A \wedge B), \Delta}\left([\mathbf{f}] \wedge^{R}\right)$

$\frac{\Gamma,[\mathrm{f}] A,[\mathrm{f}] B \Rightarrow \Delta}{\Gamma,[\mathrm{f}](A \vee B) \Rightarrow \Delta}\left([\mathrm{f}] \vee^{L}\right)$

$\frac{\Gamma \Rightarrow[\mathrm{f}] A \wedge[\mathrm{f}] B, \Delta}{\Gamma \Rightarrow[\mathrm{f}](A \vee B), \Delta}\left([\mathrm{f}] \vee^{R}\right)$

$\frac{\Gamma,[\mathrm{t}] A,[\mathrm{f}] B \Rightarrow \Delta}{\Gamma,[\mathrm{f}](A \prec B) \Rightarrow \Delta}\left([\mathrm{f}] \prec^{L}\right)$

$\frac{\Gamma \Rightarrow[\mathrm{t}] A \subseteq[\mathrm{f}] B, \Delta}{\Gamma \Rightarrow[\mathrm{f}](A \prec B), \Delta}\left([\mathrm{f}] \prec^{R}\right)$

$\frac{\Gamma,[\mathrm{t}] A,[\mathrm{f}] B \Rightarrow \Delta \Gamma,[\mathrm{f}] A,[\mathrm{t}] B \Rightarrow \Delta}{\Gamma,[\mathrm{f}](A \equiv B) \Rightarrow \Delta}\left([\mathrm{f}] \equiv^{L}\right)$

$\frac{\Gamma \Rightarrow[\mathrm{t}] A \wedge[\mathrm{f}] B,[\mathrm{f}] A \wedge[\mathrm{t}] B, \Delta}{\Gamma \Rightarrow[\mathrm{f}](A \equiv B), \Delta}\left([\mathrm{f}] \equiv^{R}\right)$

- The deduction rules for unary and binary logical connectives:

$\diamond \neg+\bullet$ : same as $\mathrm{f}+\diamond$. 


$$
\diamond \mathbf{M}+\bullet:
$$

$\Gamma,[\mathrm{t}] A,[\mathrm{t}] B \Rightarrow \Delta \Gamma,[\mathrm{t}] A,[\mathrm{~m}] B \Rightarrow \Delta \Gamma,[\mathrm{m}] A,[\mathrm{t}] B$

$\Rightarrow \Delta \Gamma,[\mathrm{m}] A,[\mathrm{~m}] B \Rightarrow \Delta$

$$
\Gamma, \mathbf{M}(A \wedge B) \Rightarrow \Delta
$$

$\left(\mathbf{M} \wedge \wedge^{L}\right)$

$\Gamma \Rightarrow[\mathrm{t}] A \triangle[\mathrm{t}] B,[\mathrm{t}] A \triangle[\mathrm{m}] B,[\mathrm{~m}] A \triangle[\mathrm{t}] B$,

$$
\begin{gathered}
{[\mathrm{m}] A \triangle[\mathrm{m}] B, \Delta} \\
\Gamma \Rightarrow \mathbf{M}(A \wedge B), \Delta \\
\Gamma,[\mathrm{t}] A,[\mathrm{t}] B \Rightarrow \Delta \Gamma,[\mathrm{t}] A,[\mathrm{~m}] B \\
\Rightarrow \Delta \Gamma,[\mathrm{t}] A,[\mathrm{f}] B \Rightarrow \Delta \\
\Gamma,[\mathrm{m}] A,[\mathrm{t}] B \Rightarrow \Delta \Gamma,[\mathrm{m}] A,[\mathrm{~m}] B \\
\Rightarrow \Delta \Gamma,[\mathrm{m}] A,[\mathrm{f}] B \Rightarrow \Delta \\
\frac{\Gamma,[\mathrm{f}] A,[\mathrm{t}] B \Rightarrow \Delta \Gamma,[\mathrm{f}] A,[\mathrm{~m}] B \Rightarrow \Delta}{\Gamma, \mathbf{M}(A \vee B) \Rightarrow \Delta}\left(\mathbf{M} \vee^{L}\right) \\
\Gamma \Rightarrow[\mathrm{t}] A \wedge[\mathrm{t}] B,[\mathrm{t}] A \triangle[\mathrm{m}] B,[\mathrm{t}] A \triangle[\mathrm{f}] B, \\
{[\mathrm{~m}] A \triangle[\mathrm{t}] B,} \\
\frac{[\mathrm{m}] A \triangle[\mathrm{m}] B,[\mathrm{~m}] A \wedge[\mathrm{f}] B,[\mathrm{f}] A \wedge[\mathrm{t}] B,[\mathrm{f}] A \triangle[\mathrm{m}] B, \Delta}{\Gamma \Rightarrow \mathbf{M}(A \vee B), \Delta}\left(\mathbf{M} \vee^{R}\right)
\end{gathered}
$$

$\Gamma,[\mathrm{t}] A,[\mathrm{t}] B \Rightarrow \Delta \Gamma,[\mathrm{t}] A,[\mathrm{~m}] B$

$\Rightarrow \Delta \Gamma,[\mathbf{f}] A,[\mathbf{f}] B \Rightarrow \Delta$

$\Gamma,[\mathrm{m}] A,[\mathrm{t}] B \Rightarrow \Delta \Gamma,[\mathrm{m}] A,[\mathrm{~m}] B$

$$
\Rightarrow \Delta \Gamma,[\mathrm{m}] A,[\mathrm{f}] B \Rightarrow \Delta
$$

$\Gamma,[\mathrm{f}] A,[\mathrm{t}] B \Rightarrow \Delta \Gamma,[\mathrm{f}] A,[\mathrm{~m}] B \Rightarrow \Delta$

$$
\frac{[\mathrm{t}] B \Rightarrow \Delta \Gamma,[\mathrm{f}] A,[\mathrm{~m}] B \Rightarrow \Delta}{\Gamma, \mathbf{M}(A \prec B) \Rightarrow \Delta}\left(\mathbf{M} \prec^{L}\right)
$$

$\Gamma \Rightarrow[\mathrm{t}] A \wedge[\mathrm{t}] B,[\mathrm{t}] A \wedge[\mathrm{m}] B,[\mathrm{f}] A \wedge[\mathrm{f}] B$,

$$
[\mathrm{m}] A \triangle[\mathrm{t}] B
$$

$\frac{[\mathrm{m}] A \triangle[\mathrm{m}] B,[\mathrm{~m}] A \triangle[\mathbf{f}] B,[\mathrm{f}] A \triangle[\mathrm{t}] B,[\mathbf{f}] A \triangle[\mathrm{m}] B, \Delta}{\Gamma \Rightarrow \mathbf{M}(A \prec B), \Delta}\left(\mathbf{M} \prec^{R}\right)$

$\Gamma,[\mathrm{t}] A,[\mathrm{t}] B \Rightarrow \Delta \Gamma,[\mathrm{t}] A,[\mathrm{~m}] B$

$$
\Rightarrow \Delta \Gamma,[\mathrm{f}] A,[\mathrm{f}] B \Rightarrow \Delta
$$

$\Gamma,[\mathrm{m}] A,[\mathrm{t}] B \Rightarrow \Delta \Gamma,[\mathrm{m}] A,[\mathrm{~m}] B$

$$
\Rightarrow \Delta \Gamma,[\mathrm{m}] A,[\mathrm{f}] B \Rightarrow \Delta
$$

$\Gamma,[\mathrm{f}] A,[\mathrm{~m}] B \Rightarrow \Delta$

$$
\Gamma, \mathbf{M}(A \equiv B) \Rightarrow \Delta
$$

$\Gamma \Rightarrow[\mathrm{t}] A \wedge[\mathrm{t}] B,[\mathrm{t}] A \wedge[\mathrm{m}] B,[\mathrm{f}] A \wedge[\mathrm{f}] B$,

$$
[\mathrm{m}] A \wedge[\mathrm{t}] B
$$

$[\mathrm{m}] A \wedge[\mathrm{m}] B,[\mathrm{~m}] A \wedge[\mathrm{f}] B,[\mathrm{f}] A \wedge[\mathrm{m}] B, \Delta$

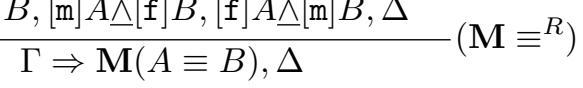

$$
\begin{aligned}
& \diamond \mathbf{L}+\bullet: \\
& \frac{\Gamma,[\mathrm{t}] A,[\mathrm{t}] B \Rightarrow \Delta}{\Gamma, \mathbf{L}(A \wedge B) \Rightarrow \Delta}\left(\mathbf{L} \wedge^{L}\right) \\
& \frac{\Gamma \Rightarrow[\mathrm{t}] A \wedge[\mathrm{t}] B, \Delta}{\Gamma \Rightarrow \mathbf{L}(A \wedge B), \Delta}\left(\mathbf{L} \wedge^{R}\right) \\
& \frac{\Gamma,[\mathrm{t}] A \Rightarrow \Delta \Gamma,[\mathrm{t}] B \Rightarrow \Delta}{\Gamma, \mathbf{L}(A \vee B) \Rightarrow \Delta}\left(\mathbf{L} \vee^{L}\right) \\
& \frac{\Gamma \Rightarrow[\mathrm{t}] A,[\mathrm{t}] B, \Delta}{\Gamma \Rightarrow \mathbf{L}(A \vee B), \Delta}\left(\mathbf{L} \vee^{R}\right) \\
& \Gamma,[\mathrm{t}] A,[\mathrm{t}] B \Rightarrow \Delta \Gamma,[\mathrm{m}] A,[\mathrm{t}] B \\
& \Rightarrow \Delta \Gamma,[\mathrm{m}] A,[\mathrm{~m}] B \Rightarrow \Delta \\
& \Gamma,[\mathrm{f}] A,[\mathrm{t}] B \Rightarrow \Delta \Gamma,[\mathrm{f}] A,[\mathrm{~m}] B \\
& \frac{\Rightarrow \Delta \Gamma,[\mathbf{f}] A,[\mathbf{f}] B \Rightarrow \Delta}{\Gamma, \mathbf{L}(A \prec B) \Rightarrow \Delta}\left(\mathbf{L} \prec^{L}\right) \\
& \Gamma \Rightarrow[\mathrm{t}] A \wedge[\mathrm{t}] B,[\mathrm{~m}] A \wedge[\mathrm{t}] B, \\
& {[\mathrm{~m}] A \wedge[\mathrm{m}] B,[\mathrm{f}] A \wedge[\mathrm{t}] B,[\mathrm{f}] A \wedge[\mathrm{m}] B,} \\
& \frac{[\mathbf{f}] A \wedge[\mathbf{f}] B, \Delta}{\Gamma \Rightarrow \mathbf{L}(A \prec B), \Delta}\left(\mathbf{L} \prec^{R}\right) \\
& \Gamma,[\mathrm{t}] A,[\mathrm{t}] B \Rightarrow \Delta \Gamma,[\mathrm{m}] A,[\mathrm{~m}] B \\
& \frac{\Rightarrow \Delta \Gamma,[\mathrm{f}] A,[\mathrm{f}] B \Rightarrow \Delta}{\Gamma, \mathbf{L}(A \equiv B) \Rightarrow \Delta}\left(\mathbf{L} \equiv^{L}\right) \\
& \frac{\Gamma \Rightarrow[\mathrm{t}] A \wedge[\mathrm{t}] B,[\mathrm{~m}] A \wedge[\mathrm{m}] B,[\mathrm{f}] A \underline{\wedge}[\mathrm{f}] B, \Delta}{\Gamma \Rightarrow \mathbf{M}(A \equiv B), \Delta}\left(\mathbf{M} \equiv{ }^{R}\right)
\end{aligned}
$$

$$
\diamond \mathbf{I}+\wedge / \vee:
$$

$\Gamma,[\mathrm{t}] A,[\mathrm{~m}] B \Rightarrow \Delta \Gamma,[\mathrm{m}] A,[\mathrm{t}] B$

$$
\frac{\Rightarrow \Delta \Gamma,[\mathrm{m}] A,[\mathrm{~m}] B \Rightarrow \Delta}{\Gamma, \mathbf{I}(A \wedge B) \Rightarrow \Delta}\left(\mathbf{I} \wedge^{L}\right)
$$

$\frac{\Gamma \Rightarrow[\mathrm{t}] A \wedge[\mathrm{m}] B,[\mathrm{~m}] A \wedge[\mathrm{t}] B,[\mathrm{~m}] A \wedge[\mathrm{m}] B, \Delta}{\Gamma \Rightarrow \mathbf{I}(A \wedge B), \Delta}\left(\mathbf{I} \wedge^{R}\right)$

$\Gamma,[\mathrm{m}] A,[\mathrm{~m}] B \Rightarrow \Delta \Gamma,[\mathrm{m}] A,[\mathrm{f}] B$ $\Rightarrow \Delta \Gamma,[\mathrm{f}] A,[\mathrm{~m}] B \Rightarrow \Delta$

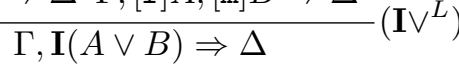

$\frac{\Gamma \Rightarrow[\mathrm{m}] A \bumpeq[\mathrm{m}] B,[\mathrm{~m}] A \wedge[\mathrm{f}] B,[\mathrm{f}] A \wedge[\mathrm{m}] B, \Delta}{\Gamma \Rightarrow \mathbf{I}(A \vee B), \Delta}\left(\mathbf{I} \vee^{R}\right)$

$\frac{\Gamma,[\mathrm{t}] A,[\mathrm{~m}] B \Rightarrow \Delta \Gamma,[\mathrm{m}] A,[\mathrm{f}] B \Rightarrow \Delta}{\Gamma, \mathbf{I}(A \prec B) \Rightarrow \Delta}\left(\mathbf{I} \prec{ }^{L}\right)$

$\frac{\Gamma \Rightarrow[\mathrm{t}] A \wedge[\mathrm{m}] B,[\mathrm{~m}] A \wedge[\mathrm{f}] B, \Delta}{\Gamma \Rightarrow \mathbf{I}(A \prec B), \Delta}\left(\mathbf{I} \prec{ }^{R}\right)$

$\Gamma,[\mathrm{t}] A,[\mathrm{~m}] B \Rightarrow \Delta \Gamma,[\mathrm{m}] A,[\mathrm{t}] B \Rightarrow \Delta \Gamma,[\mathrm{m}] A,[\mathrm{f}] B$ $\Rightarrow \Delta \Gamma,[\mathrm{f}] A,[\mathrm{~m}] B \Rightarrow \Delta$

$\Gamma, \mathbf{I}(A \equiv B) \Rightarrow \Delta$ $\left(\mathbf{I} \equiv^{L}\right)$ 


$$
\begin{aligned}
& \Gamma \Rightarrow[\mathrm{t}] A \triangle[\mathrm{m}] B,[\mathrm{~m}] A \triangle[\mathrm{t}] B,[\mathrm{~m}] A \triangle[\mathrm{f}] B, \\
& {[\mathrm{f}] A \triangle[\mathrm{m}] B, \Delta}
\end{aligned}
$$

- The deduction rules for $\underline{\Lambda} \underline{\underline{V}}$.

$$
\begin{aligned}
& \frac{\Gamma, A \Rightarrow \Delta}{\Gamma, A \_B \Rightarrow \Delta}\left(\underline{\wedge}^{L}\right)_{1} \quad \frac{\Gamma_{1} \Rightarrow A, \Delta_{1} \Gamma_{2} \Rightarrow B, \Delta_{2}}{\Gamma_{1}, \Gamma_{2} \Rightarrow A}\left(\underline{\wedge}^{R}\right) \\
& \frac{\Gamma, B \Rightarrow \Delta}{\Gamma, A \wedge B \Rightarrow \Delta}\left(\underline{\triangle}^{L}\right)_{2} \\
& \frac{\Gamma_{1}, A \Rightarrow \Delta_{1} \Gamma_{2}, B \Rightarrow \Delta_{2}}{\Gamma_{1}, \Gamma_{2}, A \underline{\bigvee} B \Rightarrow \Delta_{1}, \Delta_{2}}\left(\underline{\vee}^{L}\right) \quad \frac{\Gamma \Rightarrow A, \Delta}{\Gamma \Rightarrow A \underline{\bigvee} B, \Delta}\left(\underline{\bigvee}^{R}\right)_{1} \\
& \frac{\Gamma \Rightarrow B, \Delta}{\Gamma \Rightarrow A \underline{\vee} B, \Delta}\left(\underline{\vee}^{R}\right)_{2}
\end{aligned}
$$

Definition 3.2. $\Gamma \vdash_{\succeq_{3}} A$ if there is a sequence $\Gamma_{1} \Rightarrow$ $A_{1}, \ldots, \Gamma_{n} \Rightarrow A_{n}$ such that $\Gamma_{n} \Rightarrow A_{n}=\Gamma \Rightarrow A$, and for each $1 \leq i \leq n, \Gamma_{i} \Rightarrow A_{i}$ is deduced from the previous sequents by one of the deduction rules.

Theorem 3.3 (The soundness theorem). If $\Gamma \vdash_{\mathfrak{七}_{3}} A$ then $\models_{\mathfrak{七}_{3}} \Gamma \Rightarrow A$.

Proof. We prove that each axiom is valid and each deduction rule preserves the satisfiability.

To verify the validity of the axioms, assume that for any assignment $v, v \models \Gamma,[\mathrm{m}] p$. Then, $v \models[\mathrm{m}] p$, and so $v \models[\mathrm{m}] p, \Delta$. Similarly for other axioms.

To verify that $\left([\mathrm{m}][\mathrm{m}]^{L}\right)$ preserves the validity, assume that for any assignment $v, v \models \Gamma,[\mathrm{m}] A$ implies $v \models \Delta$. Because $v \models[\mathrm{m}] A$ implies $v \models[\mathrm{m}][\mathrm{m}] A$, for any assignment $v$, if $v \models \Gamma,[\mathrm{m}][\mathrm{m}] A$ then $v \models \Delta$. Similarly for cases of unary connectives.

To verify that $\left([\mathrm{m}] \wedge^{L}\right)$ preserves the validity, assume that for any assignment $v$,

$$
\begin{aligned}
& v \models \Gamma_{1},[\mathrm{t}] A,[\mathrm{~m}] B \text { implies } v \models \Delta_{1} ; \\
& v \models \Gamma_{2},[\mathrm{~m}] A,[\mathrm{t}] B \text { implies } v \models \Delta_{2} ; \\
& v \models \Gamma_{3},[\mathrm{~m}] A,[\mathrm{~m}] B \text { implies } v \models \Delta_{3} .
\end{aligned}
$$

For any assignment $v$, assume that $v \models \Gamma_{1}, \Gamma_{2}, \Gamma_{3},[\mathrm{~m}](A \rightarrow$ $B)$. Then,

$$
(v \models[\mathrm{t}] A \& v \models[\mathrm{m}] B),
$$

or

$$
(v \models[\mathrm{m}] A \& v \models[\mathrm{t}] B),
$$

or

$$
(v \models[\mathrm{m}] A \& v \models[\mathrm{m}] B),
$$

that is, either $v \models[\mathrm{t}] A,[\mathrm{~m}] B$, or $v \models[\mathrm{m}] A,[\mathrm{t}] B$, or $v \models$ $[\mathrm{m}] A,[\mathrm{~m}] B$. By the assumption, either $v \models \Gamma_{1},[\mathrm{t}] A,[\mathrm{~m}] B$, or $v \models \Gamma_{2},[\mathrm{~m}] A,[\mathrm{t}] B$, or $v \models \Gamma_{3},[\mathrm{~m}] A,[\mathrm{~m}] B$. By the assumption, either $v \models \Delta_{1}$, or $v \models \Delta_{2}$, or $v \models \Delta_{3}$, and $v \models \Delta_{1}, \Delta_{2}, \Delta_{3}$.

To verify that $\left([\mathrm{m}] \wedge^{R}\right)$ preserves the validity, assume that for any assignment $v, v \models \Gamma$ implies $v=[\mathrm{t}] A \triangle[\mathrm{m}] B,[\mathrm{~m}] A \wedge[\mathrm{t}] B$, $[\mathrm{m}] A \wedge[\mathrm{m}] B, \Delta$. Then, for any assignment $v$, assume that $v \models$ $\Gamma$. If $v \models \Delta$ then $v \models[\mathrm{m}](A \wedge B), \Delta$; otherwise, one of $[\mathrm{t}] A \wedge[\mathrm{m}] B,[\mathrm{~m}] A \wedge[\mathrm{t}] B$ and $[\mathrm{m}] A \wedge[\mathrm{m}] B$ is satisfied by $v$, and by the definition of the truth-value of $[\mathrm{m}](A \wedge B), v \models[\mathrm{m}](A \wedge$ $B), \Delta$.

Similarly for other cases.

\section{The completeness theorem}

Theorem 4.1 (The completeness theorem). If $\models_{\mathrm{Ł}_{3}} \Gamma \Rightarrow A$ then $\Gamma \vdash_{\mathfrak{七}_{3}} A$.

Proof. Let $\delta=\Gamma \Rightarrow A$. Define a tree, called the reduction tree for $\delta$, denoted by $T(\delta)$, from which we can obtain either a proof of $\delta$ or a show of the nonvalidity of $\delta$.

This reduction tree $T(\delta)$ for $\delta$ contains a sequent at each node, and is constructed in stages as follows.

Stage 0: $T_{0}(\delta)=\{\delta\}$.

Stage $k(k>0): T_{k}(\delta)$ is defined by cases.

Case 0 . If $\Gamma \Rightarrow \Delta$ is an axiom, write nothing above $\Gamma \Rightarrow \Delta$.

Case 1. Every topmost sequent $\Gamma \Rightarrow \Delta$ in $T_{k-1}(\delta)$ is an axiom. Then, stop.

Case 2. Not Case 1. $T_{k}(\delta)$ is defined as follows. Let $\Gamma \Rightarrow \Delta$ be any topmost sequent of the tree which has been defined by stage $k-1$.

Subcase $\left([\mathrm{t}][\mathrm{t}]^{L}\right)$. Let $[\mathrm{t}][\mathrm{t}] A_{1}, \ldots,[\mathrm{t}][\mathrm{t}] A_{n}$ be all the formulas in $\Gamma$ whose outermost logical symbol is $[\mathrm{t}][\mathrm{t}]$, and to which no reduction has been applied in previous stages. Then, write down

$$
\Gamma,[\mathrm{t}] A_{1}, \ldots,[\mathrm{t}] A_{n} \Rightarrow \Delta
$$

above $\Gamma \Rightarrow \Delta$. We say that a $\left([\mathrm{t}][\mathrm{t}]^{L}\right)$ reduction has been applied to $[\mathrm{t}][\mathrm{t}] A_{1}, \ldots,[\mathrm{t}][\mathrm{t}] A_{n}$.

Subcase $\left([\mathrm{t}][\mathrm{t}]^{R}\right)$. Let $[\mathrm{t}][\mathrm{t}] A_{1}, \ldots,[\mathrm{t}][\mathrm{t}] A_{n}$ be all the formulas in $\Delta$ whose outermost logical symbol is $[\mathrm{t}][\mathrm{t}]$, and to which no reduction has been applied in previous stages. Then, write down

$$
\Gamma \Rightarrow[\mathrm{t}] A_{1}, \ldots,[\mathrm{t}] A_{n}, \Delta
$$

above $\Gamma \Rightarrow \Delta$. We say that a $\left([\mathrm{t}][\mathrm{t}]^{R}\right)$ reduction has been applied to $[\mathrm{t}][\mathrm{t}] A_{1}, \ldots,[\mathrm{t}][\mathrm{t}] A_{n}$.

Subcase $\left(\mathbf{M L}^{L}\right)$. Let $\mathbf{M L} A_{1}, \ldots, \mathbf{M L} A_{n}$ be all the formulas in $\Gamma$ whose outermost logical symbol is $\mathbf{M L}$, and to which no 
reduction has been applied in previous stages. Then, write down

$$
\Gamma,[\mathrm{t}] A_{1}, \ldots,[\mathrm{t}] A_{n} \Rightarrow \Delta
$$

above $\Gamma \Rightarrow \Delta$. We say that a $\left(\mathbf{M L}^{L}\right)$ reduction has been applied to $\mathbf{M L} A_{1}, \ldots, \mathbf{M L} A_{n}$.

Subcase $\left(\mathbf{M L}^{R}\right)$. Let $\mathbf{M L} A_{1}, \ldots, \mathbf{M L} A_{n}$ be all the formulas in $\Delta$ whose outermost logical symbol is $\mathbf{M L}$, and to which no reduction has been applied in previous stages. Then, write down

$$
\Gamma \Rightarrow[\mathrm{t}] A_{1}, \ldots,[\mathrm{t}] A_{n}, \Delta
$$

above $\Gamma \Rightarrow \Delta$. We say that a $\left(\mathbf{M L}^{R}\right)$ reduction has been applied to $\mathbf{M L} A_{1}, \ldots, \mathbf{M L} A_{n}$.

Subcase $\left(\mathbf{L}[\mathbf{t}]^{L}\right)$. Let $\mathbf{L}[\mathbf{t}] A_{1}, \ldots, \mathbf{L}[\mathbf{t}] A_{n}$ be all the formulas in $\Gamma$ whose outermost logical symbol is $\mathbf{L}[\mathbf{t}]$, and to which no reduction has been applied in previous stages. Then, write down

$$
\Gamma,[\mathrm{t}] A_{1}, \ldots,[\mathrm{t}] A_{n} \Rightarrow \Delta
$$

above $\Gamma \Rightarrow \Delta$. We say that a $\left(\mathbf{L}[\mathrm{t}]^{L}\right)$ reduction has been applied to $\mathbf{L}[\mathrm{t}] A_{1}, \ldots, \mathbf{L}[\mathrm{t}] A_{n}$.

Subcase $\left(\mathbf{L}[\mathbf{t}]^{R}\right)$. Let $\mathbf{L}[\mathbf{t}] A_{1}, \ldots, \mathbf{L}[\mathbf{t}] A_{n}$ be all the formulas in $\Delta$ whose outermost logical symbol is $\mathbf{L}[\mathbf{t}]$, and to which no reduction has been applied in previous stages. Then, write down

$$
\Gamma \Rightarrow[\mathrm{t}] A_{1}, \ldots,[\mathrm{t}] A_{n}, \Delta
$$

above $\Gamma \Rightarrow \Delta$. We say that a $\left(\mathbf{L}[\mathrm{t}]^{R}\right)$ reduction has been applied to $\mathbf{L}[\mathrm{t}] A_{1}, \ldots, \mathbf{L}[\mathrm{t}] A_{n}$.

Similar for other cases of unary connectives.

Subcase $\left([\mathrm{m}] \wedge^{L}\right)$. Let $[\mathrm{m}]\left(A_{1} \wedge B_{1}\right), \ldots,[\mathrm{m}]\left(A_{n} \wedge B_{n}\right)$ be all the statements in $\Gamma$ whose outermost logical symbol is $[\mathrm{m}] \wedge$, and to which no reduction has been applied in previous stages by any $\left([\mathrm{m}] \wedge^{L}\right)$. Then, for each partition $\left\{I_{1}, I_{2}, I_{3}\right\}$ of $\{1, \ldots, n\}$, write down

$$
\begin{gathered}
\Gamma,[\mathrm{t}] A_{i}: i \in I_{1},[\mathrm{~m}] B_{i}: i \in I_{1} \cup I_{3},[\mathrm{~m}] A_{i}: i \in I_{2} \cup I_{3}, \\
{[\mathrm{t}] B_{i}: i \in I_{2} \Rightarrow \Delta}
\end{gathered}
$$

above $\Gamma \Rightarrow \Delta$. We say that a $\left([\mathrm{m}] \wedge^{L}\right)$ reduction has been applied to $[\mathrm{m}]\left(A_{1} \wedge B_{1}\right), \ldots,[\mathrm{m}]\left(A_{n} \wedge B_{n}\right)$.

Subcase $\left([\mathrm{m}] \wedge^{R}\right)$. Let $[\mathrm{m}]\left(A_{1} \wedge B_{1}\right), \ldots,[\mathrm{m}]\left(A_{n} \wedge B_{n}\right)$ be all the statements in $\Delta$ whose outermost logical symbol is $[\mathrm{m}] \wedge$, and to which no reduction has been applied in previous stages by any $\left([\mathrm{m}] \wedge^{R}\right)$. Then, write down

$$
\begin{aligned}
& \Gamma \Rightarrow[\mathrm{t}] A_{1} \underline{\wedge}[\mathrm{m}] B_{1},[\mathrm{~m}] A_{1} \wedge[\mathrm{t}] B_{1},[\mathrm{~m}] A_{1} \wedge[\mathrm{m}] B_{1}, \ldots, \\
& \text { [t] } A_{n} \Lambda[\mathrm{m}] B_{n},[\mathrm{~m}] A_{n} \wedge[\mathrm{t}] B_{n},[\mathrm{~m}] A_{n} \Lambda[\mathrm{m}] B_{n}, \Delta
\end{aligned}
$$

above $\Gamma \Rightarrow \Delta$. We say that a $\left([\mathrm{m}] \wedge^{R}\right)$ reduction has been applied to $[\mathrm{m}]\left(A_{1} \wedge B_{1}\right), \ldots,[\mathrm{m}]\left(A_{n} \wedge B_{n}\right)$.

Subcase $\left([\mathrm{m}] \vee^{L}\right)$. Let $[\mathrm{m}]\left(A_{1} \vee B_{1}\right), \ldots,[\mathrm{m}]\left(A_{n} \vee B_{n}\right)$ be all the statements in $\Gamma$ whose outermost logical symbol is $[\mathrm{m}] \vee$, and to which no reduction has been applied in previous stages by any $\left([\mathrm{m}] \vee^{L}\right)$. Then, for each partition $\left\{I_{1}, I_{2}, I_{3}\right\}$ of $\{1, \ldots, n\}$, write down

$$
\begin{gathered}
\Gamma,[\mathrm{m}] A_{i}: i \in I_{1} \cup I_{2},[\mathrm{~m}] B_{i}: i \in I_{1} \cup I_{3}, \\
{[\mathrm{f}] A_{i}: i \in I_{3},[\mathrm{f}] B_{i}: i \in I_{2} \Rightarrow \Delta}
\end{gathered}
$$

above $\Gamma \Rightarrow \Delta$. We say that a $\left([\mathrm{m}] \vee^{L}\right)$ reduction has been applied to $[\mathrm{m}]\left(A_{1} \vee B_{1}\right), \ldots,[\mathrm{m}]\left(A_{n} \vee B_{n}\right)$.

Subcase $\left([\mathrm{m}] \vee^{R}\right)$. Let $[\mathrm{m}]\left(A_{1} \vee B_{1}\right), \ldots,[\mathrm{m}]\left(A_{n} \vee B_{n}\right)$ be all the statements in $\Delta$ whose outermost logical symbol is $[\mathrm{m}] \mathrm{V}$, and to which no reduction has been applied in previous stages by any $\left([\mathrm{m}] \vee^{R}\right)$. Then, write down

$$
\begin{array}{r}
\Gamma \Rightarrow[\mathrm{m}] A_{1} \wedge[\mathrm{m}] B_{1},[\mathrm{~m}] A_{1} \wedge[\mathrm{f}] B_{1},[\mathrm{f}] A_{1} \wedge[\mathrm{m}] B_{1}, \ldots, \\
\left.[\mathrm{m}] A_{n} \underline{\Lambda}[\mathrm{m}] B_{n},[\mathrm{~m}] A_{n} \underline{\mathrm{f}}\right] B_{n},[\mathrm{f}] A_{n} \triangle[\mathrm{m}] B_{n}, \Delta
\end{array}
$$

above $\Gamma \Rightarrow \Delta$. We say that a $\left([\mathrm{m}] \vee^{R}\right)$ reduction has been applied to $[\mathrm{m}]\left(A_{1} \vee B_{1}\right), \ldots,[\mathrm{m}]\left(A_{n} \vee B_{n}\right)$.

Subcase $\left(\mathbf{M} \wedge \wedge^{L}\right)$. Let $\mathbf{M}\left(A_{1} \wedge B_{1}\right), \ldots, \mathbf{M}\left(A_{n} \wedge B_{n}\right)$ be all the statements in $\Gamma$ whose outermost logical symbol is $\mathbf{M} \wedge$, and to which no reduction has been applied in previous stages by any $\left(\mathbf{M} \wedge^{L}\right)$. Then, for each partition $\left\{I_{1}, I_{2}, I_{3}, I_{4}\right\}$ of $\{1, \ldots, n\}$, write down

$$
\begin{aligned}
& \Gamma,[\mathrm{t}] A_{i}: i \in I_{1} \cup I_{2},[\mathrm{t}] B_{i}: i \in I_{1} \cup I_{3}, \\
& \quad[\mathrm{~m}] A_{i}: i \in I_{3} \cup I_{4},[\mathrm{~m}] B_{i}: i \in I_{2} \cup I_{4} \Rightarrow \Delta
\end{aligned}
$$

above $\Gamma \Rightarrow \Delta$. We say that a $\left(\mathbf{M} \wedge^{L}\right)$ reduction has been applied to $\mathbf{M}\left(A_{1} \wedge B_{1}\right), \ldots, \mathbf{M}\left(A_{n} \wedge B_{n}\right)$.

Subcase $\left(\mathbf{M} \wedge \wedge^{R}\right)$. Let $\mathbf{M}\left(A_{1} \wedge B_{1}\right), \ldots, \mathbf{M}\left(A_{n} \wedge B_{n}\right)$ be all the statements in $\Delta$ whose outermost logical symbol is $\mathbf{M} \wedge$, and to which no reduction has been applied in previous stages by any $\left(\mathbf{M} \wedge \wedge^{R}\right)$. Then, write down

$$
\begin{aligned}
& \Gamma \Rightarrow[\mathrm{t}] A_{1} \underline{\wedge}[\mathrm{t}] B_{1},[\mathrm{t}] A_{1} \bumpeq[\mathrm{m}] B_{1},[\mathrm{~m}] A_{1} \bumpeq[\mathrm{t}] B_{1}, \\
& \text { [m] } A_{1} \wedge[\mathrm{m}] B_{1}, \\
& \ldots,[\mathrm{t}] A_{n} \wedge[\mathrm{t}] B_{n},[\mathrm{t}] A_{n} \wedge[\mathrm{m}] B_{n},[\mathrm{~m}] A_{n} \wedge[\mathrm{t}] B_{n},
\end{aligned}
$$




$$
[\mathrm{m}] A_{n} \Lambda[\mathrm{m}] B_{n}, \Delta
$$

above $\Gamma \Rightarrow \Delta$. We say that a $\left(\mathbf{M} \wedge^{R}\right)$ reduction has been applied to $\mathbf{M}\left(A_{1} \wedge B_{1}\right), \ldots, \mathbf{M}\left(A_{n} \wedge B_{n}\right)$.

Subcase $(\mathbf{M} \vee L)$. Let $\mathbf{M}\left(A_{1} \vee B_{1}\right), \ldots, \mathbf{M}\left(A_{n} \vee B_{n}\right)$ be all the statements in $\Gamma$ whose outermost logical symbol is $\mathbf{M} \vee$, and to which no reduction has been applied in previous stages by any $(\mathbf{M} \vee L)$. Then, for each partition $\left\{I_{1}, \ldots, I_{8}\right\}$ of $\{1, \ldots, n\}$, write down

$$
\begin{gathered}
\Gamma,[\mathrm{t}] A_{i}: i \in I_{1} \cup I_{2} \cup I_{3},[\mathrm{t}] B_{i}: i \in I_{1} \cup I_{4} \cup I_{7}, \\
{[\mathrm{~m}] A_{i}: i \in I_{4} \cup I_{5} \cup I_{6},[\mathrm{~m}] B_{i}: i \in I_{2} \cup I_{4} \cup I_{8},} \\
{[\mathrm{f}] A_{i}: i \in I_{7} \cup I_{8},[\mathrm{f}] B_{i}: i \in I_{3} \cup I_{6} \Rightarrow \Delta}
\end{gathered}
$$

above $\Gamma \Rightarrow \Delta$. We say that a $\left(\mathbf{M} \vee \vee^{L}\right)$ reduction has been applied to $\mathbf{M}\left(A_{1} \vee B_{1}\right), \ldots, \mathbf{M}\left(A_{n} \vee B_{n}\right)$.

Subcase $(\mathbf{M} \vee R)$. Let $\mathbf{M}\left(A_{1} \vee B_{1}\right), \ldots, \mathbf{M}\left(A_{n} \vee B_{n}\right)$ be all the statements in $\Delta$ whose outermost logical symbol is $\mathbf{M} \vee$, and to which no reduction has been applied in previous stages by any $\left(\mathbf{M} \vee^{R}\right)$. Then, write down

$$
\begin{aligned}
& \left.\Gamma \Rightarrow[\mathrm{t}] A_{1} \underline{\wedge} \mathrm{t}\right] B_{1},[\mathrm{t}] A_{1} \underline{\wedge}[\mathrm{m}] B_{1},[\mathrm{t}] A_{1} \underline{\wedge}[\mathrm{f}] B_{1}, \\
& {[\mathrm{~m}] A_{1} \wedge[\mathrm{t}] B_{1},[\mathrm{~m}] A_{1} \wedge[\mathrm{m}] B_{1},} \\
& \text { [m] } A_{1} \wedge[\mathrm{f}] B_{1},[\mathrm{f}] A_{1} \wedge[\mathrm{t}] B_{1},[\mathrm{f}] A_{1} \wedge[\mathrm{m}] B_{1}, \ldots, \\
& {[\mathrm{t}] A_{n} \Lambda[\mathrm{t}] B_{n},[\mathrm{t}] A_{n} \wedge[\mathrm{m}] B_{n},[\mathrm{t}] A_{n} \wedge[\mathrm{f}] B_{n},} \\
& \text { [m] } A_{n} \wedge[\mathrm{t}] B_{n},[\mathrm{~m}] A_{n} \wedge[\mathrm{m}] B_{n}, \\
& \left.[\mathrm{~m}] A_{n} \wedge[\mathrm{f}] B_{n},[\mathrm{f}] A_{n} \underline{\mathrm{t}}\right] B_{n},[\mathrm{f}] A_{n} \wedge[\mathrm{m}] B_{n}, \Delta
\end{aligned}
$$

above $\Gamma \Rightarrow \Delta$. We say that a $\left(\mathbf{M} \vee \vee^{R}\right)$ reduction has been applied to $\mathbf{M}\left(A_{1} \vee B_{1}\right), \ldots, \mathbf{M}\left(A_{n} \vee B_{n}\right)$.

Subcase $\left(\underline{\Lambda}^{L}\right)$. Let $\left(A_{1} \wedge B_{1}\right), \ldots,\left(A_{n} \wedge B_{n}\right)$ be all the statements in $\Gamma$ whose outermost logical symbol is $\Lambda$, and to which no reduction has been applied in previous stages by any $\left(\bigwedge^{L}\right)$. Then, write down

$$
\Gamma, A_{1}, B_{1}, \ldots, A_{n}, B_{n} \Rightarrow \Delta
$$

above $\Gamma \Rightarrow \Delta$. We say that a $\left(\underline{\Lambda}^{L}\right)$ reduction has been applied to $\left(A_{1} \wedge B_{1}\right), \ldots,\left(A_{n} \wedge B_{n}\right)$.

Subcase $\left(\underline{\Lambda}^{R}\right)$. Let $\left(A_{1} \underline{ } B_{1}\right), \ldots,\left(A_{n} \wedge B_{n}\right)$ be all the statements in $\Delta$ whose outermost logical symbol is $\Lambda$, and to which no reduction has been applied in previous stages by any $\left(\Lambda^{R}\right)$. Then, write down

$$
\Gamma \Rightarrow C_{1}, \ldots, C_{n}, \Delta
$$

above $\Gamma \Rightarrow \Delta$, where $C_{i} \in\left\{A_{i}, B_{i}\right\}$. We say that a $\left(\underline{\Lambda}^{R}\right)$ reduction has been applied to $\left(A_{1} \wedge B_{1}\right), \ldots,\left(A_{n} \wedge B_{n}\right)$.

Subcase $\left(\underline{\vee}^{L}\right)$. Let $\left(A_{1} \underline{\vee} B_{1}\right), \ldots,\left(A_{n} \underline{\vee} B_{n}\right)$ be all the statements in $\Gamma$ whose outermost logical symbol is $\underline{\vee}$, and to which no reduction has been applied in previous stages by any $\left(\underline{\vee}^{L}\right)$. Then, write down

$$
\Gamma, C_{1}, \ldots, C_{n} \Rightarrow \Delta
$$

above $\Gamma \Rightarrow \Delta$, where $C_{i} \in\left\{A_{i}, B_{i}\right\}$. We say that a $\left(\underline{\vee}^{L}\right)$ reduction has been applied to $\left(A_{1} \underline{\vee} B_{1}\right), \ldots,\left(A_{n} \underline{\vee} B_{n}\right)$.

Subcase $\left(\underline{\vee}^{R}\right)$. Let $\left(A_{1} \underline{\vee} B_{1}\right), \ldots,\left(A_{n} \underline{\vee} B_{n}\right)$ be all the statements in $\Delta$ whose outermost logical symbol is $\underline{\mathrm{V}}$, and to which no reduction has been applied in previous stages by any $\left(\underline{\vee}^{R}\right)$. Then, write down

$$
\Gamma \Rightarrow A_{1}, B_{1}, \ldots, A_{n}, B_{n}, \Delta
$$

above $\Gamma \Rightarrow \Delta$. We say that a $\left(\underline{\vee}^{R}\right)$ reduction has been applied to $\left(A_{1} \underline{\vee} B_{1}\right), \ldots,\left(A_{n} \underline{\vee} B_{n}\right)$.

Similar for other subcases.

So the collection of those sequents which are obtained by the above reduction process, together with the partial order obtained by this process, is the reduction tree for $\delta$, denoted by $T(\delta)$.

A sequence $\delta_{0}, \ldots$ of sequents in $T(\delta)$ is a branch if $\delta_{0}=\delta$, and for each $i, \delta_{i+1}$ is immediately above $\delta_{i}$.

Given a sequent $\delta$, if each branch of $T(\delta)$ is ended with a sequent containing an axiom, then it is a routine to construct a proof of $\delta$.

Otherwise, there is a branch $\sigma=\delta_{1}, \ldots, \delta_{n}$ of $T(\delta)$ such that there is no rule applicable for $\delta_{n}$ and $\delta_{n}=\Gamma_{n} \Rightarrow \Delta_{n}$ is not an axiom.

Let

$$
\begin{aligned}
& \cup \Gamma=\left\{\varphi: \varphi \in \Gamma_{i}, \Gamma_{i} \Rightarrow \Delta_{i} \in \sigma\right\}, \\
& \cup \Delta=\left\{\varphi: \varphi \in \Delta_{i}, \Gamma_{i} \Rightarrow \Delta_{i} \in \sigma\right\} .
\end{aligned}
$$

We define an assignment in which every formula $\varphi \in \cup \Gamma$ is true and every formula in $\varphi \in \cup \Delta$ is not.

Define $v$ such that for any propositional variable $p$,

$$
\begin{aligned}
& v(p)=\mathrm{t} \text { iff }[\mathrm{t}] p \in \cup \Gamma \\
& v(p)=\mathrm{m} \text { iff }[\mathrm{m}] p \in \cup \Gamma \\
& v(p)=\mathrm{f} \text { iff }[\mathrm{f}] p \in \cup \Gamma .
\end{aligned}
$$

By the induction on the structure of statement $A$, we prove that $v \models A$ if $A \in \cup \Gamma$ and $v \not \models A$ if $A \in \cup \Delta$.

Case $A=[\mathrm{m}]\left(A_{1} \wedge A_{2}\right) \in \cup \Gamma$. Let $\beta$ be the least-length 
segment of $\sigma$ such that $\beta=\Gamma^{\prime},[\mathrm{m}]\left(A_{1} \wedge A_{2}\right) \Rightarrow \Delta^{\prime}$ for some $\Gamma^{\prime}$ and $\Delta^{\prime}$. Then, there is a segment $\gamma$ of $\sigma$ such that $\beta$ is a segment of $\gamma$ and $\gamma$ is one of the following forms:

$$
\begin{aligned}
& \Gamma^{\prime},[\mathrm{t}] A_{1},[\mathrm{~m}] A_{2} \Rightarrow \Delta^{\prime}, \\
& \Gamma^{\prime},[\mathrm{m}] A_{1},[\mathrm{t}] A_{2} \Rightarrow \Delta^{\prime}, \\
& \Gamma,[\mathrm{m}] A_{1},[\mathrm{~m}] A_{2} \rightarrow \Delta^{\prime},
\end{aligned}
$$

say $\gamma=\Gamma^{\prime},[\mathrm{m}] A_{1},[\mathrm{~m}] A_{2} \Rightarrow \Delta^{\prime}$. By induction assumption, $v \models \Gamma^{\prime},[\mathrm{m}] A_{1},[\mathrm{~m}] A_{2}$ and $v \not \models \Delta^{\prime}$. Then, by the definition of satisfaction, $v \models \Gamma^{\prime},[\mathrm{m}]\left(A_{1} \wedge A_{2}\right)$ and $v \not \models \Delta^{\prime}$.

Case $A=[\mathrm{m}]\left(A_{1} \wedge A_{2}\right) \in \cup \Delta$. Let $\beta$ be the least-length segment of $\sigma$ such that $\beta=\Gamma^{\prime} \Rightarrow[\mathrm{m}]\left(A_{1} \wedge A_{2}\right), \Delta^{\prime}$. Then, there are segments $\gamma_{1}, \gamma_{2}, \gamma_{3}$ of $\sigma$ such that $\beta$ is a segment of $\gamma_{1}, \gamma_{2}, \gamma_{3}$ and $\gamma_{1}=\Gamma^{\prime} \Rightarrow[\mathrm{t}] A \wedge[\mathrm{m}] B, \Delta^{\prime} ; \gamma_{2}=\Gamma^{\prime} \Rightarrow[\mathrm{m}] A \wedge[\mathrm{t}] B, \Delta^{\prime}$ and $\gamma_{3}=\Gamma^{\prime} \Rightarrow[\mathrm{m}] A \wedge[\mathrm{m}] B, \Delta^{\prime}$. By the induction assumption, $v \models \Gamma^{\prime}$ and $v \not \models[\mathrm{t}] A \wedge[\mathrm{m}] B, \Delta^{\prime} ; v \not \models[\mathrm{m}] A \wedge[\mathrm{t}] B, \Delta^{\prime} ; v \not \models$ $[\mathrm{m}] A \triangle[\mathrm{m}] B, \Delta^{\prime}$, i.e., $v \not \models[\mathrm{m}]\left(A_{1} \wedge A_{2}\right), \Delta^{\prime}$.

Similarly for other cases.

This completes the proof.

\section{Conclusions}

In this paper we gave a modalized Łukasiewicz three-valued propositional logic and a Gentzen deduction system was constructed such that the soundness theorem and the completeness theorem hold in Łukasiewicz three-valued semantics of the modalized propositional logic.

In practical applications, we use the traditional fuzzy logic in which only two truth-values $t$ and $f$ are considered in the deduction, even though in semantics, a formula can have any values as the truth-values. In the Gentzen deduction system given in this paper, each truth-value contributes to the deduction, which makes the system a little clumsy. As a system which can be implemented in computer, we hope the Gentzen deduction system is used in practice in near future.

\section{Conflict of Interest}

No potential conflict of interest relevant to this article was reported.

\section{Acknowledgements}

This work was supported by the National Science Foundation of China (under grant Nos. 91224006 and 61173063) and the Ministry of Science and Technology (under grant No.201303107).

\section{References}

[1] A. Avron, "Natural 3-valued logics: characterization andproof theory," Journal of Symbolic Logic, vol. 56, no. 1, pp. 276-294, 1991. http://dx.doi.org/10.2307/2274919

[2] A. Avron, "Gentzen-type systems, resolution and tableaux," Journal of Automated Reasoning, vol. 10, no. 2, pp. 265-281,1993. http://dx.doi.org/10.1007/BF00881838

[3] D. A. Bochvar and M. Bergmann, "On a three-valued logical calculus and itsapplication to the analysis of the paradoxes of the classicalextended functional calculus," History and Philosophy ofLogic, vol. 2, no. 1-2, pp. 87112, 1981. http://dx.doi.org/10.1080/01445348108837023

[4] M. Fitting, "Many-valued modal logics II," FundamentaInformaticae, vol. 17, no. 1-2, pp. 55-73, 1992.

[5] S. Gottwald, A Treatise on Many-Valued Logics. Baldock: ResearchStudies Press, 2001.

[6] S. Gottwald, "Many-Valued Logic," Available http://plato. stanford.edu/entries/logic-manyvalued/

[7] R.Hahnle, "Advanced many-valued logics," in Handbook of Philosophical Logic, D. M. Gabbay and F. Guenthner, Eds. Dordrecht: Springer, 2001, pp. 297-395. http://dx. doi.org/10.1007/978-94-017-0452-6_5

[8] S. C. Kleene, "On notation for ordinal numbers," Journal of Symbolic Logic, vol. 3, no. 4, pp. 150-155,1938. http: //dx.doi.org/10.2307/2267778

[9] W. Li, Mathematical Logic: Foundations for InformationScience. Basel: BirkhauserVerlag AG, 2010.

[10] J. Lukasiewicz, "O logicetrojwartosciowej [On threevalued logic]," Ruch filozoficzny, vol. 5, pp. 170-171, 1920.

[11] J. Lukasiewicz, "Selected Works," in Studies in Logic and the Foundations of Mathematics, L. Borkowski, Ed. Amsterdam:North-Holland and Warsaw, 1970.

[12] E. L. Post, "Determination of all closed systems of truthtables," Bulletin American Mathematical Society, vol. 26, pp. 437, 1920.

[13] E. L. Post, "Introduction to a general theory of elementarypropositions," American Journal of Mathematics, vol. 43, no. 3, pp. 163-185, 1921. http://dx.doi.org/10.2307/ 2370324 
[14] A. Urquhart,"Basic many-valued logic," in Handbook of philosophical logic (vol. 2), D. M. Gabbay and F. Guenthner, Eds. Dordrecht: Springer, 2001, pp. 249-295. http://dx.doi.org/10.1007/978-94-017-0452-6_4

[15] W. Zhu, "Independence issues of the propositional connectivesin medium logic systems MP and MP," in AFriendly Collection of Mathematical Papers I. Changchun: Jilin University Press, 1990.

[16] J. Zhu, X. Xiao, and W. Zhu, "A survey of the developmentof medium logic calculus system and the research ofits semantics," in A Friendly Collection of Mathematical Papers I. Changchun: Jilin University Press, 1990.

[17] W. Zhu and X. Xiao, An Introduction to Foundations ofMathematics. Nanjing: Nanjing University Press, 1996.

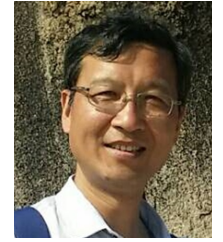

Cungen Cao received his M.S. and Ph.D. degree in software from the Institute of Mathematics, Chinese Academy of Sciences in 1989 and 1993 respectively. He is a full-time professor of ICT, CAS, since 2000. He is leading a research group of large-scale knowledge engineering and knowledge-intensive applications.

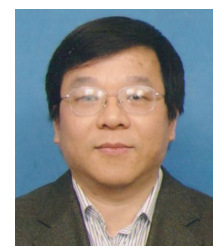

Yuefei Sui is a Professor in the Chinese Academy of Sciences (ICT), Chinese Academy of Sciences. His main interests include knowledge representation, applied logic and the theory of computability. 